\title{
RG decimation-based approach to confinement and computation on coarser lattices
}

\author{
E.T. Tomboulis* \\ Univ of California, Los Angeles \\ E-mail: tombouli@physics.ucla.edu
}

\begin{abstract}
A systematic procedure is presented for connecting short to long scales in LGT. Approximate decimations are used which can provide both upper and lower bounds on the partition function. Its exact value is then obtained by interpolation between the bounds. By iterating the procedure representations of the partition function as well as other physical quantities can be obtained on progressively coarser lattices. For $S U(2)$ IR flow into the confining strong coupling regime results for any initial $\beta$.
\end{abstract}

XXIIIrd International Symposium on Lattice Field Theory

25-30 July 2005

Trinity College, Dublin, Ireland

\footnotetext{
* Speaker.
} 


\section{Introduction}

As it is well-known, pure non-abelian gauge theory at $T=0$ and space-time dimension $d \leq 4$ possesses the extraordinary property of being in a single phase for all values of the bare gauge coupling, $0<\beta<\infty$. The theory exhibits passage from the short distance perturbative (ordered) regime to a long distance confining (disordered) regime without encountering a phase transition. Such multi-scale problems are notoriously difficult to treat from first principles. Another wellknown, and in fact somewhat similar example is the passage from laminar to turbulent flow in hydrodynamics.

In principle such problems can be treated by some systematic block-spinning or decimation procedure. Exact block-spinning schemes in gauge theories, however, so far appear intractable, both analytically and numerically. There are, however, approximate but computable decimation procedures that can provide bounds on judicially chosen quantities. Such bounds can then be used to rigorously constrain the flow behavior of the exact theory [1] - [2]. The basic procedure followed in this program is as follows:

(i) Devise decimation schemes such that at each decimation step one can obtain both an upper and a lower bound for the partition function. Furthermore, the decimations should be explicitly computable to any accuracy.

(ii) At each step interpolate between the upper and lower bound. Then the exact value of partition partition function (free energy) is obtained at some particular value of the interpolating parameter. This then gives a representation of the partition function on the decimated lattice [1].

(iii) The same procedure is then applied to the partition function in the presence of a 'twist' (external center flux).

(iv) Combining (ii) and (iii) allows consideration of appropriate long distance order parameters: the vortex free energy, Wilson loop, and Wilson lines.

We will see that the flow into the IR regime of the exact theory can be extracted without knowing the precise right numerical values of the extrapolation parameters that give the exact representations at each decimation step. For $S U(2)$, the only case explicitly considered here, the result is flow into the strong coupling confinement regime for any initial $\beta$. Furthermore, if these right numerical values of the extrapolation parameters can be explicitly obtained, one can compute appropriate quantities directly on coarser lattices.

\section{Decimation and interpolation}

Starting with some reflection positive plaquette action at lattice spacing $a$, e.g. the Wilson action $A_{p}(U)=\frac{\beta}{N} \operatorname{Re} \operatorname{tr} U_{p}$, consider the character expansion of the action exponential:

$$
F(U, a)=e^{A_{p}(U)}=\sum_{j} d_{j} F_{j}(\beta, a) \chi_{j}(U),
$$

For $\mathrm{SU}(2)$, one has $j=0, \frac{1}{2}, 1, \frac{3}{2}, \ldots, d_{j}=(2 j+1)$. It is convenient to work in terms of normalized coefficients;

$$
F(U, a)=F_{0}\left[1+\sum_{j \neq 0} c_{j}(\beta) d_{j} \chi_{j}(U)\right] \equiv F_{0} f(U, a) .
$$


The partition function on lattice $\Lambda$ is then

$$
Z_{\Lambda}(\beta)=\int d U_{\Lambda} \prod_{p} F_{p}(U, a)=F_{0}^{|\Lambda|} \int d U_{\Lambda} \prod_{p} f_{p}(U, a)
$$

We employ decimation schemes of the 'potential moving' type. The lattice is split into hypercubes of side length $\lambda a$, and plaquettes are moved from the interior of each hypercube to its boundary, where plaquette interactions are appropriately adjusted to compensate for the move. The process can be summarized as an explicit decimation rule for each step in successive decimations

$$
a \rightarrow \lambda a \rightarrow \lambda^{2} a \rightarrow \cdots \rightarrow \lambda^{n} a \quad \Longleftrightarrow \quad \Lambda \rightarrow \Lambda^{(1)} \rightarrow \Lambda^{(2)} \rightarrow \cdots \rightarrow \Lambda^{(n)},
$$

which gives explicit expressions for the computation of the Fourier coefficients at the $(m+1)$-th step given those of the $m$-th step:

$$
\begin{gathered}
F_{0}(m) \rightarrow F_{0}(m+1)=F_{0}\left(m, \zeta, r, \lambda,\left\{c_{i}(m)\right\}\right) \\
c_{j}(m) \rightarrow c_{j}(m+1)=c_{j}\left(m, \zeta, r, \lambda,\left\{c_{i}(m)\right\}\right)
\end{gathered}
$$

The rule for the decimation scheme we adopt, which need not be explicitly given here, involves parameters $\zeta, r$ which control the amount by which the interactions of the remaining plaquettes after a decimation step are renormalized to compensate for the ones that were removed.

The resulting PF after $n$ decimation steps is then:

$$
Z_{\Lambda}(\beta, n)=\prod_{m=0}^{n} F_{0}(m)^{|\Lambda| / \lambda^{m d}} \int d U_{\Lambda^{(n)}} \prod_{p} f_{p}(U, n) .
$$

It is important to note that, after each decimation step, the resulting action retains the original one-plaquette form but will, in general, contain all representations:

$$
f_{p}(U, n)=\left[1+\sum_{j \neq 0} c_{j}(n) d_{j} \chi_{j}(U)\right] \equiv \exp A_{p}(n)
$$

with

$$
A_{p}(n)=\sum_{j} \tilde{\beta}_{j}(\beta, n) \chi_{j}(U)
$$

Also, both positive and negative effective couplings $\tilde{\beta}_{j}$ will, in general, occur.

The following two basic statements can now be proved. Going from the $n-1$ step to the $n$ decimation step one has:

(I) with $\zeta=\lambda^{d-2}, \quad r=1-\varepsilon, \quad 0 \leq \varepsilon<1$ :

$$
Z_{\Lambda}(\beta, n-1) \leq Z_{\Lambda}(\beta, n)
$$

(II) with $\zeta=1, \quad r=1+\varepsilon, \quad 0 \leq \varepsilon$ :

$$
Z_{\Lambda}(\beta, n) \leq Z_{\Lambda}(\beta, n-1)
$$

In fact, in both (I), (II) one has strict inequality at any finite $\beta$. 
Translation invariance, convexity of the free energy, and reflection positivity (positivity of Fourier coefficients) underlie (I), (II).

We next interpolate between the upper and lower bounds. Introducing a parameter $\alpha,(0 \leq \alpha)$, define interpolating coefficients $\tilde{c}_{j}(n, \alpha)$ and $\tilde{F}_{0}(n, \alpha)$ such that

$$
\tilde{c}_{j}(n, \alpha)= \begin{cases}c_{j}\left(n, \zeta=\lambda^{d-2}, r=1-\varepsilon\right) & : \alpha=1 \\ c_{j}(n, \zeta=1, r=1+\varepsilon) & : \alpha=0\end{cases}
$$

and similarly

$$
\tilde{F}_{0}(n, \alpha)= \begin{cases}F_{0}\left(n, \zeta=\lambda^{d-2}, r=1-\varepsilon\right) & : \alpha=1 \\ F_{0}(n, \zeta=1, r=1+\varepsilon) & : \alpha=0\end{cases}
$$

for $0 \leq \varepsilon<1$. A variety of convenient explicit examples of such interpolating $\tilde{c}_{j}(n, \alpha), \tilde{F}_{0}(n, \alpha)$ may be given. There is, of course, nothing unique about any one such smooth interpolation. Consider more generally a family of smooth interpolations parametrized by a parameter $t$ in some interval $\left(t_{1}, t_{2}\right)$ :

$$
\tilde{c}_{j}(n, \alpha, t), \quad \tilde{F}_{0}(n, \alpha, t)
$$

Define the corresponding interpolating partition function $\tilde{Z}_{\Lambda}(\beta, n, \alpha, t)$ by the replacement

$$
F_{0}(n) \rightarrow \tilde{F}_{0}(n, \alpha, t), \quad c_{j}(n) \rightarrow \tilde{c}_{j}(n, \alpha, t)
$$

Then, for each fixed $t$, we have

$$
\tilde{Z}_{\Lambda}(\beta, n, 0, t) \leq Z_{\Lambda}(\beta, n-1) \leq \tilde{Z}_{\Lambda}(\beta, n, 1, t) .
$$

But then, by continuity, there exist a value

$$
0<\alpha=\alpha_{\Lambda}^{(n)}(t)<1
$$

such that

$$
\tilde{Z}_{\Lambda}\left(\beta, n, \alpha_{\Lambda}^{(n)}(t), t\right)=Z_{\Lambda}(\beta, n-1) .
$$

In other words, $\alpha_{\Lambda}^{(n)}(\beta, t)$ is the level surface (implicit function) solution to

$$
\tilde{Z}_{\Lambda}(\beta, n, \alpha, t)=Z_{\Lambda}(\beta, n-1) .
$$

There is thus 'parametrization invariance' in (2.10): shifts in $t$ are compensated by changes in $\alpha_{\Lambda}^{(n)}(\beta, t)$. This procedure of fixing $\alpha_{\Lambda}^{(n)}(\beta, t)$ is iterated in successive decimations $(n=1,2, \ldots)$. So starting at the original spacing $a$, after the 1 st decimation step the procedure gives an exact representation on the coarser lattice $\Lambda^{(1)}$ of the original partition function on $\Lambda$ in the form:

$$
\begin{aligned}
Z_{\Lambda}(\beta) & =F_{0}^{|\Lambda|} \int d U_{\Lambda} \prod_{p} f_{p}(U, a) \\
& =\tilde{Z}_{\Lambda}\left(\beta, \alpha_{\Lambda}^{(1)}(t), t\right) \\
& =F_{0}^{|\Lambda|} \tilde{F}_{0}\left(\alpha_{\Lambda}^{(1)}(t), t\right)^{|\Lambda| / \lambda^{d}} \int d U_{\Lambda^{(1)}} \prod_{p} f_{p}\left(U, \alpha_{\Lambda}^{(1)}(t), t\right) .
\end{aligned}
$$

Since the resulting action in (2.12) is again of the same type (2.5), iteration of the procedure results in a representation analogous to (2.12) on successively coarser lattices $\Lambda^{(n)}$.

Note that, by construction, the coefficients $\tilde{c}_{j}, \tilde{F}_{0}$ evaluated at $\alpha_{\Lambda}^{(n)}(t)$ occurring in (2.12) are always sandwiched between the explicitly computable upper bound $(\alpha=1)$ and lower bound $(\alpha=$ 0) coefficients. 


\section{Twisted partition function - Vortex free-energy}

The twisted partition function $Z_{\Lambda}^{(-)}(\beta)$ is defined as the partition function with 'twisted' action on each plaquette of a coclosed set $\mathscr{V}$ of plaquettes winding around the periodic lattice in $(d-2)$ directions perpendicular to a given 2-plane. Thus on the dual lattice $\mathscr{V}$ forms a closed 2-dim surface in $d=4$, and a closed loop in $d=3$. The 'twist' is a shift by a non-trivial element $\tau \in$ $Z(N)$ of the group center, and amounts to a discontinuous (singular) gauge transformation on the configurations in the partition function with multivaluedness in $Z(N)$, i.e. the introduction of a $\pi_{1}(S U(N) / Z(N))=Z(N)$ vortex. $\tau=-1$ for $S U(2)$.

The vortex free-energy order parameter $F_{\Lambda}^{(-)}$is then defined as:

$$
\exp -F_{\Lambda}^{(-)}=\frac{Z_{\Lambda}^{(-)}}{Z_{\Lambda}}
$$

The behavior of the Wilson loop and Wilson line correlations can be related to (3.1) through known inequalities.

There is a slight technical complication in applying the above procedure to the twisted partition function. For $Z_{\Lambda}^{(-)}$reflection positivity holds only in planes perpendicular to the directions in which the set $\mathscr{V}$ carrying the twist is winding around the lattice. One way to overcome this is to simply replace $Z_{\Lambda}^{(-)}$by the quantity $Z_{\Lambda}+Z_{\Lambda}^{(-)}$for which reflection positivity, as it is easily verified, is restored in all planes. The above development then can be carried through in this case also.

Thus, e.g., after one decimation, one has the exact representation analogous to (2.12):

$$
Z_{\Lambda}(\beta)+Z_{\Lambda}^{(-)}(\beta)=\tilde{Z}_{\Lambda}\left(\beta, \alpha_{\Lambda}^{+(1)}(t), t\right)+\tilde{Z}_{\Lambda}^{(-)}\left(\beta, \alpha_{\Lambda}^{+(1)}(t), t\right) .
$$

One may then iterate the procedure in successive decimation steps.

It should be noted that, as indicated by the notation, the $\alpha_{\Lambda}^{+(1)}(t)$ in (3.2) are fixed independently of and hence a priori can be distinct from the $\alpha_{\Lambda}^{(1)}(t)$ occurring in the representation (2.12) for the partition function $Z_{\Lambda}(\beta)$. The same of course is true for each subsequent iteration.

Consider now using these exact representations for computing the vortex free-energy (3.1). After one decimation, using (3.2), one has:

$$
\begin{aligned}
\left(1+\frac{Z_{\Lambda}^{(-)}}{Z_{\Lambda}}\right)=\frac{Z_{\Lambda}+Z_{\Lambda}^{(-)}}{Z_{\Lambda}} & =\frac{\tilde{Z}_{\Lambda}\left(\beta, \alpha_{\Lambda}^{+(1)}(t), t\right)+\tilde{Z}_{\Lambda}^{(-)}\left(\beta, \alpha_{\Lambda}^{+(1)}(t), t\right)}{Z_{\Lambda}} \\
& =\frac{\tilde{Z}_{\Lambda}\left(\alpha_{\Lambda}^{+(1)}(t), t\right)}{Z_{\Lambda}}\left[1+\frac{\tilde{Z}_{\Lambda}^{(-)}\left(\alpha_{\Lambda}^{+(1)}(t), t\right)}{\tilde{Z}_{\Lambda}\left(\alpha_{\Lambda}^{+(1)}(t), t\right)}\right] .
\end{aligned}
$$

By construction, the numerator in (3.3) is invariant under shifts in $t$; but the two factors in which it is split in (3.4) are not individually invariant. (They would be, by (2.12), only if $\alpha_{\Lambda}^{+(1)}(t)$ were to exactly coincide with $\alpha_{\Lambda}^{(1)}(t)$ for all $t$.)

One now finds that there exist a value $t=t^{*}$ at which $\tilde{Z}_{\Lambda}\left(\alpha_{\Lambda}^{+(1)}(t), t\right)$ intersects the level curve $Z_{\Lambda}=\tilde{Z}_{\Lambda}\left(\alpha_{\Lambda}^{(1)}(t), t\right)$, i.e.

$$
\tilde{Z}_{\Lambda}\left(\alpha_{\Lambda}^{+(1)}\left(t^{*}\right), t^{*}\right)=Z_{\Lambda}=\tilde{Z}_{\Lambda}\left(\alpha_{\Lambda}^{(1)}(t), t\right)
$$


Thus

$$
1+\frac{Z_{\Lambda}^{(-)}}{Z_{\Lambda}}=1+\frac{\tilde{Z}_{\Lambda}^{(-)}\left(\alpha_{\Lambda}^{+(1)}\left(t^{*}\right), t^{*}\right)}{\tilde{Z}_{\Lambda}\left(\alpha_{\Lambda}^{+(1)}\left(t^{*}\right), t^{*}\right)} .
$$

(3.5) may then be iterated in successive decimation steps. Now, by construction, the coefficients $\tilde{c}_{j}\left(n, \alpha_{\Lambda}^{+(n)}(t)\right), \tilde{F}_{0}\left(n, \alpha_{\Lambda}^{+(n)}(t), t\right)$ are sandwiched between the upper bound $(\alpha=1)$ and lower bound $(\alpha=0)$ coefficients which, for the potential-moving type of decimations employed here, are explicitly computable. In particular, the upper bound ones are essentially those of a MigdalKadanoff decimation. For $S U(2)$ this implies flow of the effective action in (3.5) into the strong coupling regime, hence confining behavior for the order parameter, for any initial $\beta$. Decimations need only be carried out till the strong coupling regime is reached. From that point onward, computations can be performed within the convergent strong coupling cluster expansion.

\section{Conclusion - Outlook}

The iterated procedure of decimation followed by interpolation between computable upper and lower bounds allows one to constrain and hence follow the qualitative RG flow of the exact theory. This is possible without knowledge of the precise numerical values of the extrapolation parameters (the $\alpha_{\Lambda}^{(n)}$ 's) needed to give the exact representation of the physical quantity (partition functions, vortex free energy, etc) considered. For $S U(2)$, the case explicitly treated, this suffices to extract IR flow into the strong coupling confinement regime for any initial $\beta$.

The derivations above only ascertain, within upper and lower bounds, the existence of appropriate values (the $\alpha^{(n)}$ 's) for the interpolating parameters reproducing a given physical quantity on successively coarser lattices. If these exact numerical values of the extrapolation parameters can be obtained, one can compute this quantity directly on coarser lattices. An advantage of the above development is that the general form of the effective action entering at each step is determined (eq. (2.5)), and only the numerical coefficients (effective couplings) are to be determined. Algorithms for doing this, involving an improved microcanonical demon technique, are under development in collaboration with A. Velytsky.

Acknowledgments: This work was partially supported by NSF-PHY-0309362.

\section{References}

[1] E.T. Tomboulis, $R G$ decimations and confinement, Nucl. Phys. B (Proc. Suppl.) 141 (2005) 115 [hep-lat/0409019].

[2] E.T. Tomboulis, From short to long scales in the QCD vacuum, Nucl. Phys. B (Proc. Suppl.) 129 (2004) 724 [hep-lat/0309006]. 